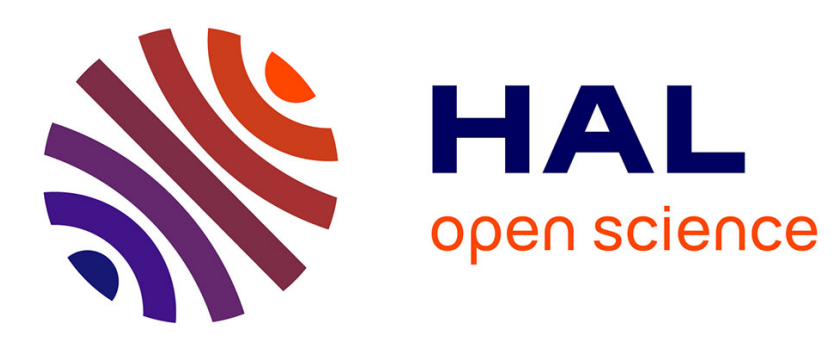

\title{
Concilier risques sanitaires liés aux sols pollués et renouvellement urbain
}

Marie-Laure Lambert

\section{To cite this version:}

Marie-Laure Lambert. Concilier risques sanitaires liés aux sols pollués et renouvellement urbain. Droit et ville, 2014, 78, pp.97-108. hal-01227192

\section{HAL Id: hal-01227192 \\ https://hal.science/hal-01227192}

Submitted on 10 Nov 2015

HAL is a multi-disciplinary open access archive for the deposit and dissemination of scientific research documents, whether they are published or not. The documents may come from teaching and research institutions in France or abroad, or from public or private research centers.
L'archive ouverte pluridisciplinaire HAL, est destinée au dépôt et à la diffusion de documents scientifiques de niveau recherche, publiés ou non, émanant des établissements d'enseignement et de recherche français ou étrangers, des laboratoires publics ou privés. 


\title{
Concilier risques sanitaires liés aux sols pollués et renouvellement urbain
}

\author{
Marie-Laure LAMBERT \\ Maître de conférences à l'Université d'Aix-Marseille
}

Le droit français du renouvellement urbain ou de la reconstruction sur sites pollués n'en finit pas d'évoluer par àcoups.

Depuis les premières circulaires de 1993, en passant par la loi Bachelot de 2003, l'ordonnance de 2010 qui a créé au Code de l'environnement une nouvelle "police des sols pollués», et aujourd'hui la loi ALUR, on sent bien que le législateur pare au plus pressé, harcelé par les jurisprudences nombreuses, inquiété par le nombre de sites pollués en France (4540 sites BASOL et près de 300000 sites BASIAS) et le nombre de constructions déjà recensées, ou à venir, sur ces sites (917 écoles sont construites en France sur d'anciens sites industriels) et surtout contraint par la baisse des financements publics, qui touche notamment le budget de l'ADEME.

L'article 173 de la loi ALUR actualise en quelque sorte cette accumulation de cahots juridiques, de dispositions additives sur la gestion des sites et sols pollués. Poursuivant l'effort du Grenelle sur la diffusion de l'information sur la pollution des sols, il introduit surtout la possibilité pour des tiers aménageurs de prendre en charge financièrement et techniquement la réhabilitation d'un site pollué. Sans vouloir décrire de façon exhaustive ces innovations juridiques, il sera ici question d'identifier une logique de double transfert qui se manifeste dans ce texte : le transfert de la charge de la dépollution des industriels pollueurs vers les tiers aménageurs (I), et le transfert du contrôle de l'Etat sur les risques sanitaires liés à la construction sur sites pollués vers des bureaux d'études privés, les communes, voire vers les propriétaires eux-mêmes (II). 


\section{LA PROTECTION JURIDIQUE DES INDUSTRIELS AU DÉTRIMENT DE L'ENVIRONNEMENT ET DE LA SANTÉ PUBLIQUE}

L'évolution du droit français de la réhabilitation des sites et sols pollués (SSP) est marquée par un abandon progressif des exigences de protection de l'environnement, qui marquait les premiers textes. Pourtant, le Code de l'environnement pose des principes d'action clairs: «les milieux naturels sont un patrimoine commun, leur restauration, leur remise en état sont d'intérêt général et s'inspirent du principe pollueur-payeur» (L. 110-1 C. env.). Il est vrai qu'en s'opposant au projet de directive européenne sur la protection des sols, et en excluant ces derniers de la liste des milieux physiques du livre II du Code de l'environnement, lequel ne fait référence qu'à l'eau et l'air, les politiques publiques françaises ont renoncé à considérer le sol comme un milieu naturel digne de préservation, comme une ressource qui se raréfie et devrait être protégée, voire remise en état. Ainsi le droit français des SSP s'éloigne-t-il à la fois de l'objectif de restauration ou de remise en état des sols (A), mais également du principe pollueur-payeur (B).

\section{A. UN CHANGEMENT DE FORMULATION QUI MARQUE UNE REGRESSION : DE LA REMISE EN ETAT A LA REHABILITATION}

En matière de SSP, l'objectif de protection de l'environnement et de la santé publique, qui était clairement inscrit dans les premiers textes ${ }^{1}$, en application du principe «pollueur-payeur» a été atténué par le passage assumé par le ministère de l'environnement à un «principe de réalité » tenant compte des coûts importants des opérations de dépollution des sites (bilan coûts/avantages), d'autant qu'une partie importante de la charge de ces dépollutions pèse sur l'ADEME, en charge des sites orphelins.

Cette évolution a été progressive, depuis l'introduction de la réhabilitation en fonction de l'usage futur du site, d'abord dans les circulaires du 7 juin 1996 et du 10 décembre 1999, puis dans

\footnotetext{
${ }^{1}$ Version de 1994 de l'art. 34-1 du décret de 1977: «Lorsque l'ICPE est mise à l'arrêt définitif, son exploitant remet son site dans un état tel qu'il ne s'y manifeste aucun des dangers ou inconvénients mentionnés à l'article $1^{\circ}$ de la loi du 19 juillet 1976. Le préfet peut à tout moment imposer à l'exploitant les prescriptions relatives à la remise en état du site, par arrêté ».
} 
la loi «Bachelot» du 30 juillet $2003^{2}$, qui donne une valeur législative à la notion.

Depuis l'adoption de cette loi, mais surtout du décret du 13 septembre $2005^{3}$, les obligations de dépollution des anciens exploitants industriels ont été singulièrement allégées par la généralisation de la réhabilitation en vue d'un usage futur industriel $^{4}$, qui nécessite les travaux de remédiation les plus légers. En outre, le décret dégage l'ancien exploitant de toute obligation de dépollution supplémentaire dans le cas d'un changement d'usage ultérieur (R. 512-39-4) et l'arrêt du Conseil d'Etat «Alusuisse» de $2005^{5}$ a éteint la responsabilité de l'ancien exploitant au bout de trente ans. Sachant enfin que le plan de réhabilitation du site doit se fonder sur le calcul financier du bilan coûts/avantages, on comprend que les travaux seront menés seulement si leur coût n'est pas excessif. La doctrine considère donc à juste titre que la « logique de traitement des SSP est très bienveillante pour les exploitants industriels $»^{6}$.

Aussi assiste-t-on dans la loi ALUR à un changement de formulation qui donne forme à ce renoncement. Il ne s'agit plus, désormais, de remettre en état les sites, mais de les réhabiliter, c'est-à-dire les rendre aptes à un usage défini. Or, si cette formulation est conforme à la pratique française dont on sait qu'elle ne correspond ni à une remise à l'état initial du site, ni à une dépollution complète, elle semble en contradiction avec les objectifs posés par la directive IED ${ }^{7}$.

En effet, devant l'échec de l'adoption du projet de directive «sols », le législateur européen a néanmoins réussi à intégrer certaines obligations de protection du sol par les industriels, dans le cadre de la directive IED de 2010 relative aux émissions industrielles. Pas moins de quatre considérants et neuf articles de cette directive affirment l'importance de limiter les pollutions du

\footnotetext{
2 loi n²003-699, du 30 juillet 2003, relative à la prévention des risques technologiques et naturels et à la réparation des dommages

3 décret n²005-1170 du 13 septembre 2005 relatif aux conditions de cessation d'activité des ICPE

${ }^{4}$ L'usage futur industriel est retenu pour toutes les ICPE fermées avant le 1/10/05 (art. 34-5 du décret) ou pour les ICPE fermées après le 1/10/05, dans les cas où la négociation entre le maire et l'exploitant échoue, ou si le maire ne parvient pas à prouver l'incompatibilité avec les documents d'urbanisme.

${ }^{5}$ Conseil d'Etat, 8 juillet 2005, Société Alusuisse-Lonza-France.

${ }^{6}$ F-G. Trébulle « Sols pollués : le clair-obscur de la loi ALUR », Envt et DD, août-sept 2014, p. 13-18, p. 17.

7 Directive IED 2010/75/UE du 24 novembre 2010 relative aux émissions industrielles, qui remplace la directive IPPC depuis 2013.
} 
$\mathrm{sol}^{8}$, dans le cadre du principe du pollueur-payeur. En outre, l'article 3-2 définit utilement la pollution du sol comme «l'introduction directe ou indirecte, par l'activité humaine, de substances, de vibrations, de chaleur ou de bruit dans l'air, l'eau ou le sol, susceptibles de porter atteinte à la santé humaine ou à la qualité de l'environnement, d'entraîner des détériorations des biens matériels, une détérioration ou une entrave à l'agrément de l'environnement ou à d'autres utilisations légitimes de ce dernier ». On constate que les intérêts protégés sont assez larges puisqu'il peut s'agir d'atteintes à la santé humaine, même si elles ne sont pas «graves», ou d'atteintes à la «qualité de l'environnement», voire au simple «agrément de l'environnement ». En outre, la directive dispose que le droit applicable aux sites industriels doit comporter «des prescriptions appropriées garantissant la protection du sol» (article 14), une surveillance périodique au moins une fois tous les dix ans pour le sol (art. 16), un rapport de base contenant les informations nécessaires pour déterminer le niveau de contamination du sol, de manière à effectuer une comparaison quantitative avec l'état du site lors de la cessation définitive des activités (art. 22). Enfin, «lors de la cessation définitive des activités, (...) si l'installation est responsable d'une pollution significative du sol (...) par des substances dangereuses pertinentes par rapport à l'état constaté dans le rapport de base(...), l'exploitant prend les mesures nécessaires afin de remédier à cette pollution, de manière à remettre le site dans cet état. »

Ainsi, l'exploitant, selon le droit communautaire, est effectivement contraint à remettre le site dans l'état initial où il se trouvait lorsqu'il a commencé son activité. Même si les pollutions qui lui sont dues étaient autorisées avant l'entrée en vigueur de la directive, il doit également les «éliminer, maîtriser, confiner ou réduire » pour éviter qu'elles ne présentent «un risque important pour la santé humaine ou pour l'environnement », compte tenu de l'usage prévu du site?

\footnotetext{
${ }^{8}$ Considérants 12, 23, 24, 25, et articles $1^{\circ}, 3,14,16,22,46,52,57,64$.

9 Art 22-3 : «Sans préjudice du premier alinéa, lors de la cessation définitive des activités, si la contamination du sol et des eaux souterraines sur le site présente un risque important pour la santé humaine ou pour l'environnement, en raison des activités autorisées exercées par l'exploitant avant que l'autorisation relative à l'installation ait été mise à jour pour la première fois après le 7 janvier 2013, et compte tenu de l'état du site de l'installation constaté conformément à l'article 12, paragraphe 1, point d), l'exploitant prend les mesures nécessaires visant à éliminer, maîtriser, confiner ou réduire les substances dangereuses pertinentes, de sorte que le site, compte tenu de
} 
Or, les articles L. 515-30 et L. 515-31 du Code de l'environnement, qui transposent cette obligation, manquent singulièrement de précision : s'ils reprennent l'obligation de réaliser le rapport de base, ils renvoient à un décret à venir le soin de définir les «conditions dans lesquelles le site est remis en état et dans lesquelles il peut être tenu compte de la faisabilité technique des mesures de remise en état ». On peut ici mesurer l'empressement des autorités françaises à appliquer ces dispositions de la directive IED.

Ainsi, si les formulations reprises dans la loi ALUR correspondent bien à la pratique française qui n'exige ni remise à l'état initial des sols pollués, ni dépollution au regard de normes de qualité génériques qui puissent être généralisées à tous les sites industriels, il n'est pas certain qu'elles ne puissent être sanctionnées pour non conformité aux objectifs fixés par la directive IED.

Enfin, la nouvelle rédaction de 1'article L. 556-3 réduit la police des sols, qui avait été créée par l'ordonnance du 17 décembre $2010^{10}$, et qui concernait initialement toute «pollution des sols ou de risque de pollution des sols », en ne qualifiant finalement de pollutions des sols que celles qui «présentant des risques pour la santé, la sécurité, la salubrité publiques et l'environnement au regard de l'usage pris en compte». On retombe ici encore sur la pratique française d'une remédiation en fonction des seuls usages futurs du sol.

\section{B. LE TRANSFERT DES OBLIGATIONS DE DEPOLLUTION A UN TIERS}

Surtout, la loi ALUR ose aller jusqu'au bout de la logique du dispositif français de gestion des sites pollués, en proposant de confier la réhabilitation d'un site pollué à un « tiers payeur » qui porterait un projet d'aménagement ou de construction sur ce site. Ce tiers viendrait donc se substituer au responsable initial de la pollution, améliorant à peine le glissement qui s'observait depuis des années, du principe «pollueur-payeur», au principe «contribuable-payeur». Il est vrai que, dans l'objectif de reconvertir rapidement d'anciennes friches industrielles insérées

son utilisation actuelle ou de l'utilisation qu'il a été convenu de lui donner à l'avenir, cesse de représenter un tel risque ».

10 Ordonnance n 2010-1579 du 17 décembre 2010 portant diverses dispositions d'adaptation au droit de l'Union européenne dans le domaine des déchets. 
dans le milieu urbain, afin de reconstruire la ville sur elle-même dans un souci de densification, il devenait urgent de trouver des financeurs solvables capables de prendre en charge la réhabilitation de ces sites, sans trop grever les finances publiques (bien que l'ADEME intervienne en soutien financier la plupart du temps). Des indications techniques sur les modalités de construction sur ces SSP ont d'ailleurs été publiées récemment pour compléter le dispositif juridique ${ }^{11}$.

Ce tiers payeur sera certes utile dans les cas de pollutions historiques où le responsable d'un site abandonné depuis des décennies a disparu depuis longtemps. Le ministère indique en effet qu'environ $70 \%$ du marché financier de la dépollution (études et travaux) se réalise sur des projets d'aménagement, notamment en zone urbaine, qui ne sont pas adossés à la cessation d'activité d'une ICPE. En revanche, le dispositif est critiquable dans la mesure où il pourrait permettre, dans certains cas, d'évacuer la responsabilité juridique et financière d'un exploitant encore existant et solvable.

La loi ALUR précise ainsi le dispositif français, qui peut être résumé comme suit : le droit des ICPE prévoit une réhabilitation par l'ancien exploitant a minima, c'est-à-dire en fonction d'un usage futur qui sera la plupart du temps défini comme industriel, nécessitant très peu de travaux de dépollution ${ }^{12}$. Une fois que cette mise en sécurité peu coûteuse est effectuée ${ }^{13}$, on passe à une deuxième phase, prévue par les articles L. 556-1, 2 et 3, qui transfèrent les travaux de dépollution à un tiers. L'article L. 5561 concerne le changement d'usage ultérieur d'un ancien site ICPE, qui sera réhabilité par un tiers. L'article L. 556-2 prévoit également les cas de construction ou lotissement par un tiers investisseur sur un site inscrit en secteur d'information, qu'il s'agisse d'une ancienne ICPE ou pas.

Si l'objectif de trouver de nouveaux financeurs pour dépolluer des sites en vue d'un projet immobilier est intéressant, l'article L.

\footnotetext{
${ }^{11}$ BRGM, Guide relatif aux mesures constructives utilisables dans le domaine des SSP, 11 septembre 2014 - BRGM http://www.developpementdurable.gouv.fr/spip.php?page $=$ doc\&id_article $=40742$

12 Voir M.-L. Lambert «L'après Métaleurop, l'effritement des ambitions initiales de la loi Bachelot », Droit et Ville ${ }^{\circ}$ 65/2008, p. 239-259.

${ }^{13}$ Articles L. 512-6-1, L. 512-7-6 et L. 512-12-1 Code environnement.
} 
512-21 nouveau pêche par son manque de clarté dans ses objectifs et sa rédaction. ${ }^{14}$

D'une part, l'intervention du tiers peut intervenir dès la fermeture de l'ICPE, il est donc parfaitement possible que ce tiers endosse immédiatement la responsabilité de la dépollution, en lieu et place d'un ancien exploitant pourtant solvable, qui serait ainsi totalement à l'abri du principe pollueur-payeur. Dans ce cas de figure, on peut estimer peu équitable de laisser à la charge des futurs occupants du projet immobilier (puisque le prix d'achat des logements intègrera le coût de la dépollution du site) ou de la collectivité (ADEME, EPF ou commune qui aiderait financièrement ce projet) le coût d'une dépollution dont le responsable est pourtant connu et identifié.

D'autre part, la syntaxe de cet article est étrangement polysémique: lorsqu'il est écrit qu' «un tiers intéressé peut demander au représentant de l'Etat dans le département de se substituer », on doit bien sûr entendre que le tiers demande à pouvoir lui-même se substituer, mais l'on peut entendre aussi que le tiers demande au représentant de l'Etat de se substituer. Certes, l'interprétation est osée, mais dans un domaine aussi conflictuel que celui des sols pollués, il serait préférable de bannir ce genre d'approximation. D'autant que les premières versions du projet de loi étaient plus claires : "lors de la cessation d'activité d'une installation classée pour la protection de l'environnement, le préfet peut prescrire à un tiers qui en fait la demande, (...) la réalisation des mesures de remise en état pour l'usage envisagé par le demandeur ». A tel point que l'on peut se demander si l'abandon de cette rédaction initiale pour une rédaction plus ambiguë est réellement le fruit d'une maladresse.

\section{UNE TENDANCE À L'ABANDON DES CONTRÔLES DE L'ÉTAT SUR LES RISQUES SANITAIRES}

Il est également important de noter que les obligations imposées à ce tiers en matière de vérification des risques sanitaires liées à la construction sur sites pollués seront moins contrôlées, en tout cas par les services de l'Etat.

\footnotetext{
${ }^{14}$ Article L. 512-21 (nouveau) - Lors de la mise à l'arrêt définitif d'une ICPE ou postérieurement, un tiers intéressé peut demander au représentant de l'Etat dans le département de se substituer à l'exploitant, avec son accord, pour réaliser les travaux de réhabilitation en fonction de l'usage que ce tiers envisage.
} 


\section{A. LE TRANSFERT DE LA POLICE SANITAIRE DES SSP AU MAIRE ET AUX BUREAUX D'ETUDES PRIVES}

Le ministère de l'environnement considère qu'en tout état de cause, « la responsabilité de la maîtrise des risques incombe au maître d'ouvrage au titre du Code civil. Il lui revient ainsi de réaliser ses aménagements dans les règles de l'art du domaine, présentés dans l'annexe 2 de la note ministérielle du 8 février 2007, qui s'appliquent pleinement à de telles situations $»^{15}$.

C'est peut-être pourquoi la loi ALUR réalise un basculement dans le domaine de la police sanitaire des sols pollués: ce contrôle, qui reste pourtant une mission régalienne de l'Etat, censé assurer la salubrité publique, est insensiblement transféré par la loi ALUR à d'autres acteurs.

D'une part, même sur le terrain d'une ancienne ICPE, lors d'un changement d'usage ultérieur, la surveillance des risques sanitaires et des normes de dépollution ne sera pas effectuée par les services de la DREAL, mais par un bureau d'études privé. Cette privatisation du contrôle, déjà observée dans le contrôle des ICPE soumises à déclaration ou à enregistrement se poursuit ici, en violation du principe posé par le Conseil d'Etat, selon lequel le service de la police «ne saurait être confié qu'à des agents placés sous l'autorité directe de l'administration ${ }^{16}$. Ainsi l'article L. 556-1 prévoit-il que le maître d'ouvrage doit définir lui-même les mesures de gestion de la pollution des sols et les mettre en œuvre. Il "fait attester de cette mise en ouvre par un bureau d'études certifié dans le domaine des sites et sols pollués, conformément à une norme définie par arrêté du ministre chargé de l'environnement, ou équivalent ». S'il demeure une pollution résiduelle sur le terrain concerné, le maître d'ouvrage en informe le propriétaire et le préfet qui peut créer sur le terrain concerné un secteur d'information sur les sols. Enfin, en cas de modification du projet initial, le maître d'ouvrage complète ou adapte luimême, si nécessaire, les mesures de gestion de la pollution.

Il est donc clair que l'Etat se dégage ici de ses missions de surveillance des risques sanitaires liés à la reconstruction sur sites pollués.

15 Site du ministère de l'environnement: http://www.developpementdurable.gouv.fr/Les-limites-de-la-legislation-sur.html

${ }^{16}$ Conseil d'Etat, Assemblée, 17 juin 1932, Ville de Castelnaudary, requête numéro 12045, rec. p. 595. 
D'autre part, pour les sites pollués ne relevant pas du cadre des installations classées, le ministère considère ici encore que «le rôle de l'Etat n'apparaît pas pouvoir aller au-delà des recommandations, sauf à ce qu'un péril imminent et avéré conduise l'autorité préfectorale à devoir se substituer au maire de la commune, compétent en matière de police générale de salubrité. Le propriétaire d'un site a toutefois, sur le plan civil, une responsabilité quant aux dommages que son site pourrait causer à autrui $»^{17}$.

La tendance se manifeste donc depuis 2010 de laisser au maire la responsabilité d'effectuer la police des opérations de construction sur sites pollués hors ICPE. De ce point de vue, la loi ALUR n'a pas été au bout de la clarification nécessaire sur l'autorité compétente en matière de "police des sols » prévue à l'article L. 556-3. On avait pu croire innocemment que la précédente version de l'art L. 556- $1^{18}$ créait une nouvelle police des sols, générale et assez audacieuse, qui permettait de remédier à toute pollution des sols, avec une compétence possible du maire. Si l'on sait depuis le décret du 2 janvier 2013 (R. 556-1 C. env.) que l'autorité compétente, en cas de sols pollués par des ICPE, reste le préfet, rien n'est précisé dans le cas où les sols sont pollués par d'autres sources. Le maire est donc potentiellement l'autorité désignée pour assurer et assumer la police des sols pollués par des déchets, des pollutions diffuses dont la source est peu identifiée, voire dans le cas de pollutions historiques générées par des établissements non classés ou par des ICPE dont la responsabilité de l'exploitant sera éteinte en vertu du délai de trente ans ${ }^{19}$. L'article L. 556-2 issu de la loi ALUR ne précise pas non plus son rôle dans la vérification des risques sanitaires en matière de reconstruction sur site pollué. Il y est seulement indiqué que «les projets de construction ou de lotissement prévus dans un secteur d'information sur les sols font l'objet d'une étude des sols», et que «pour les projets soumis à permis de construire ou d'aménager, le maître d'ouvrage fournit dans le dossier de demande de permis une attestation garantissant la réalisation de

\footnotetext{
17 http://www.developpement-durable.gouv.fr/La-loi-du-30-juillet-2003-et1.html. Voir aussi le guide de l'aménageur, qui met l'accent sur la communication nécessaire de la part de l'aménageur, pour assurer «l'acceptabilité du projet d'aménagement sur site pollué (usage futur, servitudes éventuelles, teneur en polluants restant sur le site).»: http://www.developpement-durable.gouv.fr/-Guide-de-l-amenageur,6529.html

${ }^{18}$ Voir M.-L. Lambert et T. Schellenberger «L'absence de protection des sols et du sous-sol dans les lois Grenelle », in S. Dupouy (dir.), Jalons pour une économie verte, PUAM, 2012, p. 333.

${ }^{19}$ CE Alusuisse Lonza précité.
} 
cette étude des sols et de sa prise en compte dans la conception du projet de construction ou de lotissement. Cette attestation doit être établie par un bureau d'études certifié dans le domaine des sites et sols pollués ». Il reste sous-entendu que l'autorité qui délivre le permis de construire endosse ainsi la responsabilité de vérifier cette attestation.

La police des risques sanitaires liés à la construction sur sites pollués est donc transférée, explicitement ou pas, au maire et aux bureaux d'études privés. Est, par suite, transférée à ces mêmes acteurs la responsabilité en cas de dommages sanitaires qui se révèleraient au bout de quelques années parmi les occupants de ces immeubles construits sur des sites pollués.

Les risques juridiques pour les communes, en matière de sites pollués, ont donc augmenté en proportion du désinvestissement des anciens exploitants industriels ${ }^{20}$ : incertitudes juridiques liées aux régimes différents (ICPE, déchets, pollutions des sols hors ICPE) ; possibilité de se voir imposer un usage futur industriel par l'ancien exploitant, qui maîtrise la négociation ; possibilité de se voir imposer des choix d'urbanisme par le préfet (détermination de l'usage futur, prise en compte de la pollution des sols, servitudes et secteurs d'information); risque de devoir payer le surcoût de la dépollution d'un site en cas de changement d'usage ultérieur ou sur un site historique (pollué depuis plus de trente ans); responsabilité de devoir effectuer la police de la réhabilitation et de la reconstruction sur site pollué.

\section{B. LE TRANSFERT DE L'OBLIGATION DE VIGILANCE A D'AUTRES ACTEURS}

Les nouvelles dispositions de la loi ALUR prévoient en outre une information élargie sur les nouveaux «secteurs d'information sur les sols »(L. 125-6. C. env.). Sur ces terrains dont la pollution est connue (vraisemblablement ceux inscrits dans la banque BASOL, mais un décret en déterminera les critères), devront être réalisées des études de sols et des «mesures de gestion de la pollution pour préserver la sécurité, la santé ou la salubrité publiques et l'environnement », notamment en cas de changement d'usage.

\footnotetext{
${ }^{20}$ Voir sur ce point Amélie Guillier - Urbanisme et sols pollués : quel rôle du maire dans la gestion du passif industriel ?, Prix spécial du GRIDAUH 2012, téléchargeable: http://www.gridauh.fr/theses-et-memoires/prix-dememoire-du-gridauh/liste-des-candidats-primes/
} 
C'est surtout le degré d'opposabilité de ces secteurs d'information qui intrigue. Matérialisés par des documents graphiques annexés aux documents d'urbanisme, ils n'auront que peu de valeur normative et ne seront pas opposables aux autorisations d'urbanisme ${ }^{21}$. En revanche, cette information génère une conséquence importante en limitant la marge de manœuvre, déjà faible, du maire dans la détermination de l'usage futur du site, et donc des exigences de dépollution. On rappellera en effet que, dans la procédure de délimitation de l'usage futur du site, et donc des travaux de dépollution à effectuer par l'ancien exploitant, le maire ne peut négocier un usage sensible (usage urbain ou création d'une école), permettant d'imposer à l'industriel une dépollution plus poussée, que s'il arrive à démontrer que l'usage prévu (industriel) est incompatible avec les documents d'urbanisme (L. 512-6-1 et R. 512-39-2-III et IV C. env.). Or, le fait d'introduire a priori dans ces documents d'urbanisme la prise en compte de la pollution des sols empêche finalement le maire de prévoir un usage urbain ou sensible, puisqu'il devrait tenir compte de l'état ou des risques de pollution des sols. La nouvelle rédaction de l'article L. 125-6 C.Env. issue de la loi ALUR, dans cette optique, est moins claire que la précédente version issue de la loi Grenelle 2 qui imposait explicitement au maire de prendre en compte les informations sur les risques de pollution des sols dans les documents d'urbanisme lors de leur élaboration et de leur révision. Mais ce dispositif, sous couvert de transparence, nous semble très pernicieux au regard des blocages qu'il peut favoriser lors des négociations entre maire et ancien exploitant, en vue de la détermination de l'usage futur du site.

Enfin, la loi ALUR renforce l'information du public. D'une part, l'Etat publie les informations aujourd'hui contenues dans la banque BASIAS, par le biais d'une carte des anciens sites industriels et activités de services. D'autre part, le certificat d'urbanisme indique si le terrain est situé sur un site répertorié sur cette carte.

Ces dispositions vont certes dans le sens d'un renforcement de l'information du public sur les risques environnementaux, dans la droite ligne de ce qui a été fait avec la mise en place du «géoportail » en matière de risques naturels et technologiques ${ }^{22}$.

\footnotetext{
${ }^{21}$ Voir L. Balzano et C. Billmann, Les apports de la loi «ALUR » dans le domaine des sites et sols pollués, RDI n ${ }^{\circ}$ 9, sept. 2014, p. 432-441.

${ }^{22} \mathrm{http}: / /$ www.geoportail.gouv.fr
} 
Pour autant, on peut craindre que ce transfert de l'information en direction des personnes exposées aux risques ne manifeste un glissement progressif de l'obligation de prévention des risques de l'Etat vers une responsabilisation de la « victime informée ».

Le mécanisme est le même avec le renforcement de l'obligation d'information de l'acquéreur ou du locataire (L. 125-7 C. env.). On peut d'ailleurs se demander si la nouvelle rédaction de cet article issue de la loi ALUR constitue réellement un renforcement, puisqu'il modifie étrangement la sanction de l'inobservation de l'information. Qu'il s'agisse des terrains occupés par des ICPE (L. 514-20) ou de la pollution historique des terrains situés en secteur d'information sur les sols (L. 1257), la possibilité d'obtenir l'annulation de la vente ou une restitution du prix est limitée, non plus à la découverte d'une pollution cachée, mais à la découverte d'une pollution qui rendrait «le terrain impropre à sa destination précisée dans le contrat ». Il s'agit là d'une protection du vendeur ${ }^{23}$. En revanche, le délai d'action de l'acheteur (ou du locataire) est également modifié, puisqu'il est limité à deux ans, mais ce délai ne court qu'à compter de la découverte de la pollution, disposition favorable à l'acheteur.

In fine, on peut se demander si ces dispositions ne marquent pas également un autre transfert de l'obligation de vigilance vers l'acheteur d'un terrain, notamment si on les interprète à la lumière de la responsabilité possible du «propriétaire négligent », que codifie l'article L. 556-3-II- $2^{\circ}$, étendant par là même la jurisprudence Wattelez ${ }^{24}$. Ainsi, à titre subsidiaire, en cas de défaillance des responsables de premier rang, devient responsable «le propriétaire de l'assise foncière des sols pollués par une activité ou des déchets s'il est démontré qu'il a fait preuve de négligence ».

La mise en évidence de ces approximations, et des libertés qu'ont pris le législateur et le pouvoir réglementaire au regard du principe pollueur-payeur, au nom d'un principe de réalité qui favorise trop évidemment les exploitants industriels, au détriment du contribuable, des riverains et habitants des sites pollués, conduit à espérer que vienne le temps d'une réflexion poussée et fondée sur l'intérêt général qui permette de construire un droit

\footnotetext{
${ }^{23}$ Même si elle peut être compensée par l'application du droit commun de la garantie des vices cachés. Voir F.-G. Trebulle p. 16.

${ }^{24}$ CE 26 juillet 2011 Wattelez.
} 
français, et plus tard communautaire, de la remise en état et de la protection des sols ${ }^{25}$.

${ }^{25}$ Voir le projet cliniquedudroitdessols.fr. 